\title{
Forming and Manipulation of Structures of Metallic Microparticles in Suspension in External Alternating Electric Field
}

\begin{abstract}
M. ZIENTARA ${ }^{a, *}$ AND D. JAKUBCZYK ${ }^{b}$
${ }^{a}$ pSci Marcin Zientara, Wąwozowa 33 lok. 4, PL-02796 Warsaw, Poland

${ }^{b}$ Institute of Physics, Polish Academy of Sciences, Aleja Lotnikow 32/46, PL-02668 Warsaw, Poland

(Received October 31, 2018; revised version September 25, 2019; in final form September 26, 2019)

Metallic microparticles, suspended in a dielectric liquid in external alternating electric field, tend to organise into regular structures. Since the structures depend on the external field parameters, they enable testing the general properties of complex systems, as well as engineering new (meta)materials. In our experiments on Cu microparticles suspended in a mixture of rapeseed oil and $n$-hexadecane in AC field, four system states (structure types) were distinguished and described: (i) driven gas state, (ii) vertical chains, (iii) fully randomly oriented chains, and (iv) percolation state. The states can be rapidly switched by manipulating the amplitude and the frequency of the AC field. The states exhibiting higher and lower order occur alternatingly versus a one-field-parameter scan. Two interstate transitions, involving microstructure reorganisation, were discussed in detail: (1) driven gas-vertical chains state transition and (2) randomly oriented chains-percolation state transition. A simple model of interactions, utilising the concept of a local electric field and considering charges and dipoles interactions, was proposed to explain the driven gas-vertical chains state transition. The transition was found independent of the external field amplitude, and the transition frequency was found to be only on dependent material properties. A simple formula predicting the transition frequency was obtained. The randomly oriented chains/percolation network transition was discussed in detail. The transition position for external field frequency and amplitude was found unequivocal, though a significant formation/disintegration hysteresis was observed versus both these parameters. The transition was found to be weakly dependent on the microparticle charge and the network formation is expected to be controlled by ohmic current. Thus, a description in the language of electric circuits was proposed for future development.
\end{abstract}

DOI: 10.12693/APhysPolA.136.866

PACS/topics: field-driven assembly, metallic microparticles suspension, microparticles structures, percolation transition

\section{Introduction}

Dispersions exposed to external fields harbour many remarkable phenomena, such as freezing by heating [1] or line formation [2]. These systems may serve as test plots for fundamental research concerning the nature of complexity or the nature and properties of interactions in complex systems, e.g., dynamic behaviour of granular media or distribution of particles on interfaces [3-8]. Investigations point to a class of interactions and phenomena that determine the behaviour of such systems. Depending on the physical and chemical properties of the suspension components, electrostatic and magnetostatic interactions [9-11], electrophoresis [12], electroosmosis [13], dielectrophoresis [14, 15], as well as dipolar interactions and electrohydrodynamic effects $[8,16,17]$ have been observed. The size of the system and the configuration of external fields influence the system as well $[12,18]$. The formation of many interesting structures has been observed in suspensions of metallic microparticles, including static vertical chains [11],

*corresponding author; e-mail: marcin.zientara@psci.eu honeycomb structures, the Wigner crystals, toroidal vortices, "pulsating rings" [19], "two-tones", "Saturn-rings", and "flowers" [10]. This kind of suspension also constitutes an easy-to-handle platform (e.g. with digital video microscopy [20]) for diverse experiments, yielding vast material for analysis (see, e.g., [9-11, 19-21]).

This work concerns a non-equilibrium, open-dynamic system of metallic microparticles moving in an external, spatially uniform, and time-periodic electric field (EEF) and suspended in a dielectric liquid. The EEF lines are vertical (locally parallel to the gravitational field lines) and the top electrode is insulated, permitting no direct conduction path in the liquid. The details of the experimental setup are given in Sect. 2.

We have found that this system has four states of different properties, associated with strikingly different $2 \mathrm{D}$ and 3D microparticle structures. The states of the system can be switched by manipulating the amplitude and the frequency of the EEF. In two of the states, the particles organise into ordered, static structures in the form of a regular set of vertical chains or in the form of a percolated network. In two others, the particles either behave as a (driven) gas or form a random set of fully randomly oriented chains. The states exhibiting higher and lower order occur alternatingly versus a one-parameter scan. 
We have found that the ordered structures form due to the interactions of charges and dipoles (induced in particles by an EEF), strongly assisted by field-driven individual particle oscillations, as well as due to the local flow of ohmic current. Since the competition/cooperation among the various interactions is a function of EEF parameters, potentially a variety of structures could be formed. In this work, we concentrated on two of them.

We propose a model that correctly describes the conditions for the occurrence of the vertical chains state, thus enabling the prediction of the corresponding EEF parameters. As a consequence, we provide a tool for designing such structures for a given type of metallic particles.

We also present a preliminary analysis of the percolated, conducting network formed by all the particles in the suspension. It seems to indicate that this structure could be described in the framework of electric circuit theory in the context of minimal conductivity.

The far-reaching motivation for this work was the fabrication of new optical meta-materials with tailored optical properties (scattering, refraction) [23-25]. After scaling-down, methods from soft-matter physics based on field-driven assembly, might replace lithography [26].

\section{Experimental setup and procedures}

A suspension of copper powder (nearly spherical grains, average radius of $\sim 20 \mu \mathrm{m}$ ) in a mixture of rapeseed (canola) oil and $n$-hexadecane was placed inside a capacitor driven with an alternative current (AC). The particles were cleaned of oxide contaminations and the suspension was carefully degassed (under vacuum) before the experiments to suppress bubbles formation.

The capacitor was built of two ITO electrodes and of the chamber holding the suspension, placed between them (Fig. 1). The distance between the electrodes was $h=2.75 \mathrm{~mm}$. The top electrode was isolated, in order to prevent contact charge electrophoresis (CCEP) particle-mediated transport of charges between the electrodes. The bottom electrode was in contact with the suspension, in order to enable efficient charging of the suspended particles. The chamber was made of polycarbonate (Macrolon, BASF). It was $10 \mathrm{~mm}$ in diameter and $2 \mathrm{~mm}$ high.

$N$-hexadecane constituted 13.5 vol.\% of the mixture. In this way the (relative) permittivity of liquid (dielectric constant) $\epsilon_{r}$ of the mixture was adjusted to that of polycarbonate (2.96), which guaranteed uniformity of the electric field inside the capacitor and suppressed sticking of the charged particles to the chamber walls. The dielectric parameters were obtained from [27-29] and the Maxwell-Garnett approximation was utilised. Both rapeseed oil and $n$-hexadecane are only weakly polar. However, the rapeseed oil, which dominates in the mixture, exhibits a significant polarisability of $\approx 3 \mathrm{pF}(\mathrm{pm})^{3}[28]$.

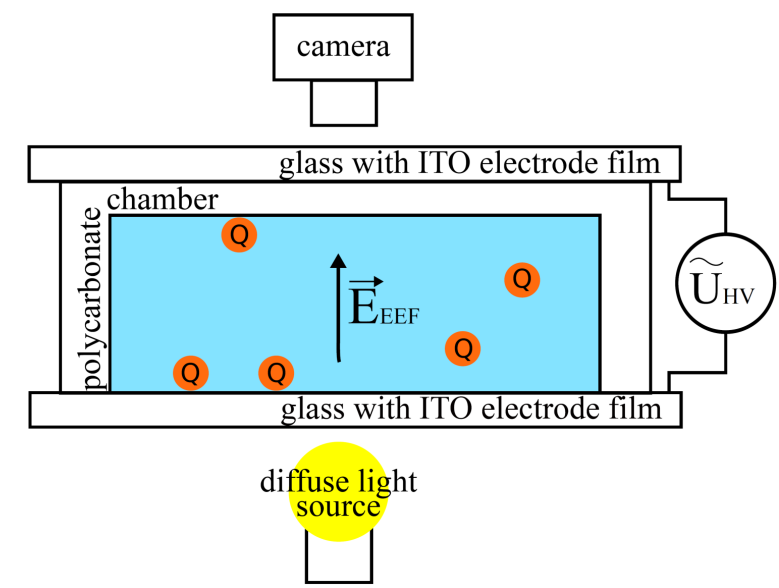

Fig. 1. The experimental setup schematic view.

The electrodes were driven with sinusoidal AC high voltage $(\mathrm{HV})$ of the peak amplitude $U_{0}$ from 0.2 to $7 \mathrm{kV}$, from the amplifier (TREK 10/10B), driven in turn by the signal generator (TABOR WW5062). The chamber was monitored through the microscope nearly from the top $\left(\approx 3^{\circ}\right.$ tilt $)$. Since the optical system was focused just below the top electrode, such arrangement allowed some assessment of vertical structures. The system evolution could be recorded with the camera. A sample recording can be found in [30].

The initial charging/detachment of microparticles from the lower electrode (chamber floor) was achieved by quick reversing of the HV applied between the electrodes. Due to polarisation/screening effects in the dielectric liquid, the available static field strength was not enough to lift the microparticles from the chamber floor. However, due to their tiny (point-like) ohmic contact with the bottom electrode, their recharging time was larger than the polarisation reversal in the dielectric and they were dragged upwards by the reorienting molecules/dipoles. When the HV was reversed slowly, the effect was not observed.

In order to suppress the influence of microparticles sedimentation, the system was "annealed" with low frequency field $(f \leq 1 \mathrm{~Hz}$ ) for 5 min to uniformly charge and distribute the suspended particles before each measurement. It should be pointed out that the MaxwellWagner charge relaxation time is negligible for a suspension of metallic particles (compare [31]). We expect however that the average imposed particle charge was a function of "annealing field" amplitude.

The peak amplitude $\left|E_{\mathrm{EEF}}\right|=U_{0} /\left(\epsilon_{r} h\right)$ and the frequency $f$ of the applied electric field were the parameters of the experiment. These parameters were scanned in two ways: (i) the frequency was scanned at constant amplitude or (ii) the amplitude was scanned at constant frequency.

In the first mode of experiment (constant field amplitude), the amplitude of the annealing field was usually different than during the scan. So first, the EEF ampli- 
tude was set at a desired value and next the frequency of the field was scanned from $1 \mathrm{~Hz}$ up to some end value. In the second mode (constant field frequency), the frequency and amplitude of annealing field were always different than during the scan. So first, the frequency was increased to a desired value (usually few hundred $\mathrm{Hz}$ ) and the amplitude was reduced to $0.25 \mathrm{kV}$ and next the amplitude was scanned up to some end value.

\section{Discussion of experimental results}

As it has been briefly mentioned above, during the parameter scan the system was passing through four different dynamical and structural states (see Fig. 2 and Fig. 3 and compare [30] — note that the driven gas and the vertical chains states are rather short-lived). All of them manifest in the first mode of scan, in the following order versus the increasing frequency: (i) the driven gas state, (ii) the vertical chains, (iii) the fully randomly oriented chains, and (iv) the percolation state. In the second mode of scan, the driven gas state and the vertical chains state were inaccessible when the used frequency was too high.

We found that the state of the system depends on the parameters value rather than on the mode in which it was reached. In other words, the final state of the system evolution was identical in the both modes. However, some hysteresis was observed.

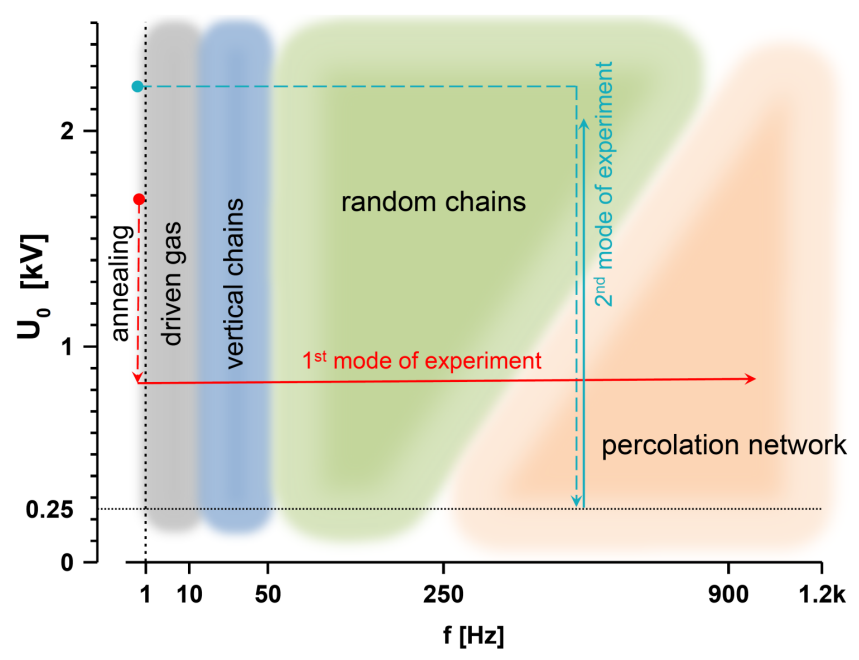

Fig. 2. A qualitative "phase diagram" of the system states. The abscissa is in a square-root scale. The state creation/destruction hysteresis is not shown. The transitions between states are rather diffuse: the transition between the driven gas and the vertical chains state is discussed in Sect. 3.2, while the positions of random chains-percolation network transition are shown in Fig. 4. The concept of both modes of experiment is shown as red and green arrowed sample traces. The dashed lines correspond to startingpoint preparation, while the solid lines correspond to one-parameter scan.

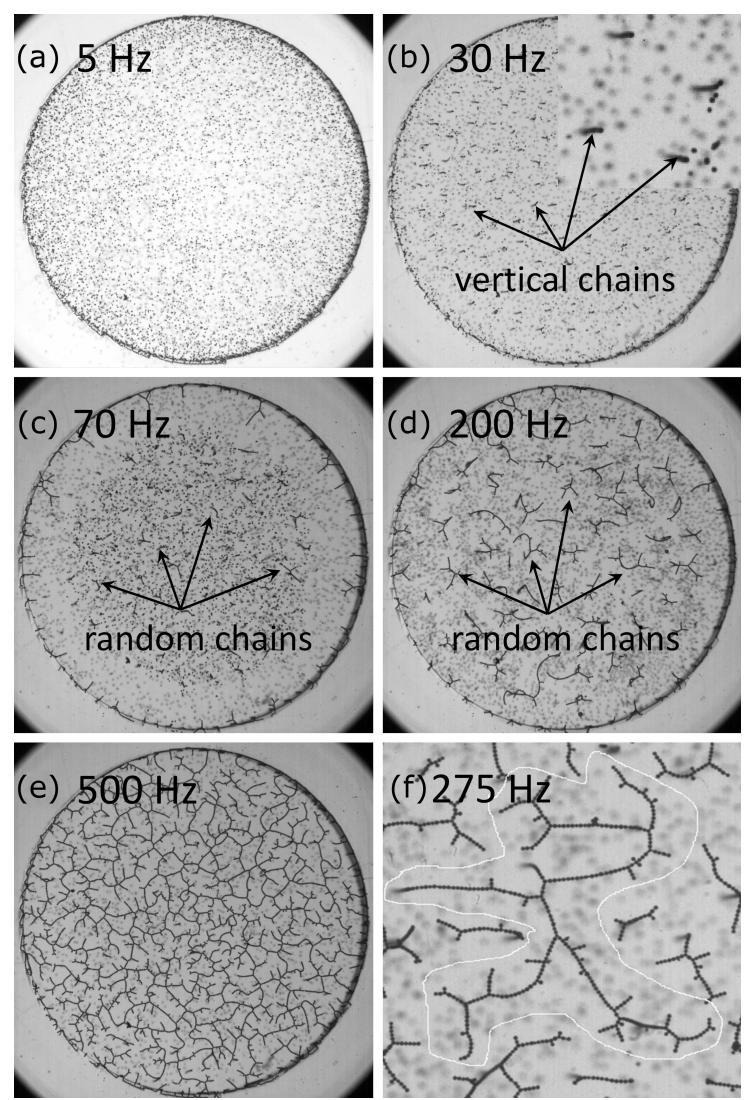

Fig. 3. A sequence of the system states observed during the frequency scan at constant amplitude: $U_{0}=1.1 \mathrm{kV}$ (first mode of experiment). The corresponding frequencies are given in parts: (a) the driven gas state, (b) the vertical chains, (c,d) the fully randomly oriented chains and (e) the percolation state. A (complex) chain initiating the percolation transition is shown (surrounded by white a line) in part (f) and a magnified view of vertical chains is shown in inset in part (b).

\subsection{The driven gas state}

The driven gas was the first observed state of the system during the experiment performed with the first mode. In this state, the motion of the particles is dominated by simple electrophoresis - non-collective oscillatory motion driven by direct interaction of their charges with the uniform (low frequency, i.e., $<10 \mathrm{~Hz}$ ) EEF. The frequency-dependent amplitude $A_{\mathrm{EEF}}$ of individual particle oscillations can be derived from the equation of motion of an individual charged particle in suspension, interacting with EEF in over-damping mode [2]:

$$
A_{\mathrm{EEF}}=\frac{2 Q\left|E_{\mathrm{EEF}}\right|}{\omega \zeta},
$$

where $\omega=2 \pi f$ is the angular frequency, $Q$ is the effective (screened) particle charge, and $\zeta$ is the friction constant of the suspension, comprising particle size and dispersion medium viscosity. 


\subsection{The vertical chains state}

The driven gas state is relatively short-lived for the parameters range. Already for the EEF frequency slightly above $10 \mathrm{~Hz}$, the transition to the vertical chains state occurs (Fig. 3b), regardless of the EEF amplitude. In the vertical chains state (quite typical for a set of dipolar particles which can move freely in the ordering field), particles aggregate into chains along the EEF lines, though small fluctuations of individual particles interacting with EEF are still visible. We expect these residual motions to be responsible for the stability of the chains. Individual particle fluctuations prevent permanent contact between the particles, thus suppressing the charge transport between the particles and along the chains to the uninsulated electrode. We show below that the vertical chains form as the result of energy minimisation mainly between dipole-dipole and EEF-dipole interactions.

In order to study in greater detail the vertical chains state in a system consisting of charged metal particles in dielectric liquid exposed to an external AC field, we propose a quite general, simple model of interactions in the system. It enables to estimate conditions at which the chains appear and are stable. The formation of such structures in other particles/liquid/external field configurations was reported, e.g., in [11, 14, 15, 22, 32, 33].

A single particle experiences the so-called local electric field (LEF). LEF consists of EEF and of the fields generated by a few nearest neighbours (the suspension concentration was relatively low). In order to pin-point the transition, we consider only the particle charge and the electrical dipole induced in the particle by EEF (higher multipoles are not considered due to low EEF frequency). Thus LEF can be expressed as

$$
\boldsymbol{E}_{\mathrm{loc}}=\sum_{i} \boldsymbol{E}_{Q_{i}}+\sum_{i} \boldsymbol{E}_{p_{i}}+\boldsymbol{E}_{\mathrm{EEF}},
$$

where subscripts $Q$ and $p$ stand for charge and dipole respectively, while $i$ enumerates the particle neighbours. Consequently, the interaction energy of a particle consists of a series of terms

$$
\begin{aligned}
& W=\sum_{i}\left(W_{Q, Q_{i}}+W_{p, Q_{i}}+W_{Q, p_{i}}+W_{p, p_{i}}\right) \\
& \quad+W_{Q, \mathrm{EEF}}+W_{p, \mathrm{EEF}},
\end{aligned}
$$

where the first subscript pertains to the particle and the second to the interacting field (source). The significance of particular terms is a function of the state of the system. Studying the experimental results allowed us to identify the terms significant for the transition between the driven gas state and the vertical chains state. As the result this transition can be characterised with a much simplified forms of Eqs. (2) and (3).

It can be reasonably assumed that the part of LEF generated by a fairly symmetrical structure of charges, on average, cancels out: $\sum_{i} \boldsymbol{E}_{Q_{i}}=0$. Further on, it is evident that the dipole-dipole interaction dominates the chargedipole interaction at close distance [32]. In effect, only the terms which describe interactions of the dipole with LEF and the charge with EEF remain.

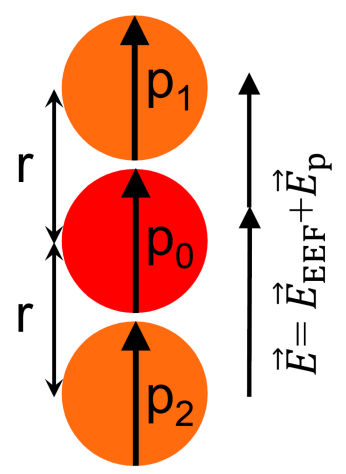

Fig. 4. The 3-particle elementary cell of the vertical chain oriented along the field. It should be noted that the particles are not in galvanic contact.

In general, LEF can be non-uniform near the particle. This distortion can come only from interaction between the particles and would lead to dielectrophoreticlike effects. However, since (i) EEF is the main component of LEF and it is uniform in our setup, and (ii) the dipole-dipole interaction is spatially symmetrical and so the time average of the associated electric field is also uniform, it is reasonable to neglect the LEF distortion. Obviously, this assumption does not hold for a setup of electrodes, where EEF is not uniform [12, 14, 15].

Since we are primarily interested in the final stage of the state transition, we considered a minimal system necessary for the formation of vertical chains. The system is composed of three particles, lined up along EEF. An $N$-particle chain, is composed of $N-2$ overlapping triads. This simplification is justified by experimental observations (compare Fig. 3b and Fig. 4). The total energy of the dipole interactions can be expressed [34]:

$W_{p}=\sum_{i \neq j} W_{p_{i}, p_{j}}+\sum_{i} W_{p_{i}, \mathrm{EEF}}=-\frac{1}{2}\left(\boldsymbol{p} \boldsymbol{E}_{\mathrm{loc}}^{*}+\boldsymbol{p}^{*} \boldsymbol{E}_{\mathrm{loc}}\right)$,

where $i, j=1,2,3$ and

$$
\boldsymbol{p}=\alpha \boldsymbol{E}_{\mathrm{loc}}
$$

is the electric dipole moment of the particle. The particle polarizability can be expressed as [34]:

$$
\alpha=N \frac{e^{2}}{m_{e}} \frac{1}{\omega_{0}^{2}+\mathrm{i} \gamma \omega},
$$

where $N$ is the number of elementary charges per particle, $\omega_{0}$ and $\gamma=N \gamma_{e}$ are the eigenfrequency and the dumping factor of charge oscillation in the particle, respectively, $\gamma_{e}$ is the dumping factor for electron [34], and $m_{e}$ and $e$ are the mass and the charge of electron, respectively - note $Q=N e$. On the other hand, LEF reduces to

$$
\boldsymbol{E}_{\mathrm{loc}}=\boldsymbol{E}_{p}+\boldsymbol{E}_{\mathrm{EEF}},
$$

where $\boldsymbol{E}_{p}$ is the field, acting on the central particle, generated by the two neighbouring dipoles (compare Fig. 4):

$$
\boldsymbol{E}_{p}=\frac{1}{\pi \epsilon_{0} \epsilon_{r}} \frac{\boldsymbol{p}}{(2 a)^{3}},
$$


where $a$ is the particle radius and $\epsilon_{0}$ is the permittivity of vacuum. Introducing Eqs. (5)-(8) into Eq. (4) and neglecting terms of the order of $a^{-6}$ leads to a compact formula for the energy of interaction between a particle and its two neighbours

$$
W_{p}=-\frac{1}{2} \boldsymbol{E}_{\mathrm{EEF}}^{2}\left[1+\frac{\alpha+\alpha^{*}}{\pi \epsilon_{0} \epsilon_{r}(2 a)^{3}}\right]\left(\alpha+\alpha^{*}\right) .
$$

As it has been already mentioned, it can be reasonably assumed that the chain ordering is controlled by dipole-dipole interaction and activated by charge-EEF interaction. This is analogous to a sub-system immersed in a thermal bath characterised with the energy of $k_{B} T[32]$. The energy of the "thermal bath" — a charged particle in EEF - can be estimated as the work done by EEF in one oscillation period (making use of Eq. (1)):

$$
\Delta W_{Q, \mathrm{EEF}}=\frac{\pi Q^{2}\left|E_{\mathrm{EEF}}\right|^{2}}{\omega \zeta}=\frac{\pi}{2} Q\left|E_{\mathrm{EEF}}\right| A_{\mathrm{EEF}} .
$$

Thus, the ratio between the interaction energy and the activation energy can be written as

$$
\begin{aligned}
& R(\omega)=\frac{W_{p}}{\Delta W_{Q, E E F}}=\left(-\frac{\omega \zeta}{\pi N m_{e}}\right) \times \\
& {\left[1+\frac{N e^{2}}{4 \pi \epsilon_{0} \epsilon_{r} a^{3} m_{e}} \frac{\omega_{0}^{2}}{\omega_{0}^{4}+\gamma^{2} \omega^{2}}\right] \frac{\omega_{0}^{2}}{\omega_{0}^{4}+\gamma^{2} \omega^{2}} .}
\end{aligned}
$$

We expect that its maximum $R_{\max }(\omega)$ corresponds to the vertical chain formation. Its location versus frequency can be determined (see Appendix) as

$$
\omega\left(R_{\max }\right)=0.22 \frac{\sigma}{\epsilon_{0} \epsilon_{r}(2 a)^{3} n},
$$

where $n$ is the concentration of free charges and $\sigma$ is the resistivity of particle material, respectively, and all irrelevant factors were combined into one numerical value. It can be readily noticed that the position of the maximum does not depend on the particle charge and, in consequence, neither on EEF amplitude. It depends only on the particle radius and on the material properties of particle and medium, which were all constant in our experiments. For the parameters of our experiments, Eq. (12) yields the transition frequency $f_{t}=13.3 \mathrm{~Hz}$, while in the experiments the transition from the driven gas state to the vertical chains state was observed between 11 and $15 \mathrm{~Hz}$. A reasonably good agreement signifies that considering only the dipole-dipole interaction with the two nearest neighbours in the chain is sufficient to describe the reordering process associated with the studied transition.

\subsection{The randomly oriented chains}

The vertical chains persisted versus frequency up to $\approx 50 \mathrm{~Hz}$ when, as we expect, the flow of ohmic current along the chains destroyed the EEF-induced dipoles. The next state, encountered in both modes of experimental scans, was the randomly oriented chains state (Figs. 3c and d). It is characterised by random motion of individual particles and randomly appearing, randomly oriented, unstable chains. We expect that dipole-dipole, charge-charge, and charge-dipole interactions play equal roles then. This state persisted until one or two of the chains, which were connected to the bottom electrode, became stable (Fig. 3f). This was happening for frequency $>250 \mathrm{~Hz}$. The first few stable chains served as the condensation nuclei for the forming structure.

\subsection{The percolation state}

The transition initiated in this manner, invariably led to a $2 \mathrm{D}$ percolation network forming a maximal (fractal) cluster directly under the whole top (insulated) electrode (Fig. 3e). The network remained connected to the bottom electrode via several chains. Thus we expect that an ohmic current flowed through the whole network, and that the network formation was current controlled. Such possibility is indicated by $[35,36]$. In Ref. [36] it was shown that in a system with co-planar electrodes the formation of net-like structures was associated with the conductivity of particles. This implies that the description of interactions, which we adopted for the driven gas/vertical chains state, is insufficient for the percolation state. Instead this, the network could be described as a system of randomly connected electric circuits and the percolation transition associated with minimal energy dissipation/circuit impedance. Similarly, the disintegration of the network, during the return scan, could be associated with increasing energy dissipation. We intend to provide a detailed description in the language of electrical network analysis in a separate paper.

A similar 2D net-like structure was observed in a system, which consisted of polystyrene particles suspended in an electrolyte [18]. The structure appeared as the result of the competition between the repulsive dipoledipole interaction and the attractive electrohydrodynamic interaction. 3D percolation structures are typical for systems of dipolar particles without external fields [37-39]. Applying a strong enough external field breaks down the structure and the system evolves towards an assemble of chains oriented along the external field [39].

The percolation network formation/disintegration events are presented in Fig. 5 versus the EEF parameters: the frequency $f$ and the amplitude represented by $U_{0}$, for both types of parameter scan. The data points pertaining to a given annealing field amplitude (corresponding to the driving voltage amplitude $U_{a}$ ) are connected with the eye-guiding lines (annealing field frequency was fixed at $1 \mathrm{~Hz}$ for all cases). The $\left(f, U_{0}\right)$ position of each event was reproducible for the given annealing parameters, though the observed formation/disintegration hysteresis versus both $f$ and $U_{0}$ was significant. Since the branches corresponding to different annealing field amplitudes differ, it can be inferred that the network formation/disintegration is also (weakly) controlled by the particle charge $Q$ imposed by the annealing process.

Increasing EEF frequency above the transition frequency $f_{p}$ was not changing the configuration of 

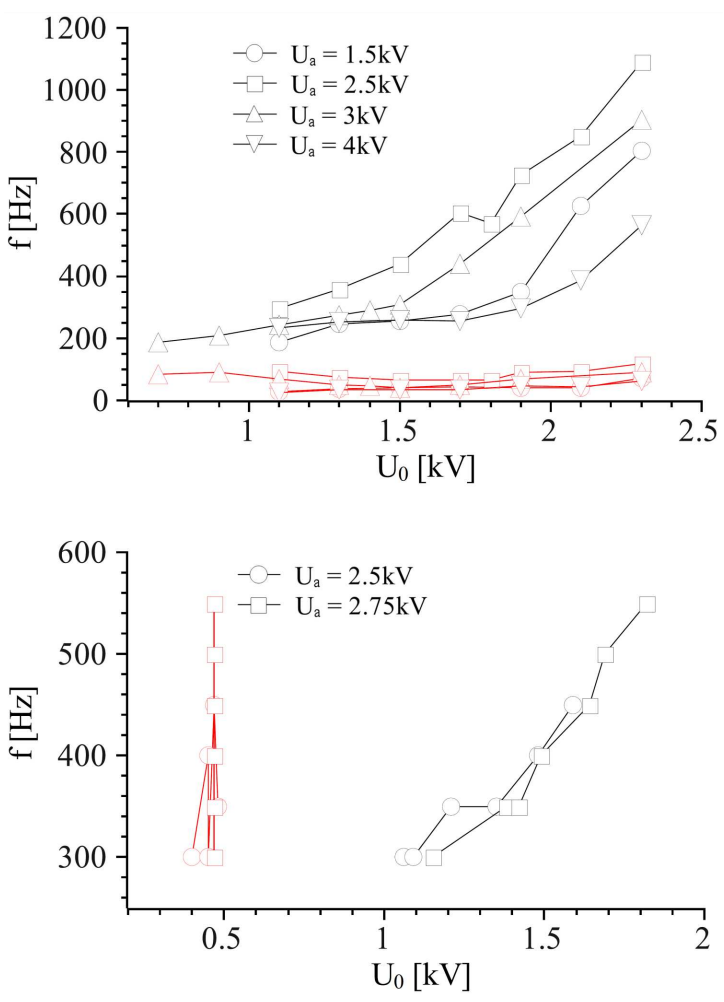

Fig. 5. The percolation network formation/disintegration events presented versus EEF parameters: the frequency $f$ and the amplitude represented by $U_{0}$, for the both types of parameter scan. Top part: the frequency scanned at a fixed amplitude. Bottom part: the amplitude scanned at a fixed frequency. The data points pertaining to a given annealing voltage amplitude $U_{a}$ (frequency fixed at $1 \mathrm{~Hz}$ for all cases) are connected with the eye-guiding lines. The black points correspond to the percolation network formation, while the red points correspond to the network disintegration.

the percolation network. However, for $f \gtrsim 900 \mathrm{~Hz}$ we observed a significant release of heat, ultimately leading to boiling of the liquid, which obviously terminated the experimental scan. As it has been mentioned above, the disintegration of the network during the return scan was observed at a frequency $f_{d}$ much lower than $f_{p}$ (Fig. 5). Repetitive scanning over this frequency range induced regular formation/disintegration of the percolation network. The same scenario was encountered for the second mode of parameter scan.

It is worth noticing that the region corresponding to the stable network structure in the first (fixed amplitude) mode of the parameter scan (above the $f\left(U_{0}\right)$ curve), corresponds to the randomly oriented chains state for the second (fixed frequency) mode of the parameter scan and vice versa. The $f\left(U_{0}\right)$ curve constitutes the common (narrow) region of the network existence for the both methods of the parameter scan. This implies that putting the system on the $f\left(U_{0}\right)$ curve guaranties transformation to the percolation network state.

\section{Conclusions}

We observed several interesting physical phenomena concerning assembly of microstructures in a fairly simple system of metallic microparticles in suspension in externally applied AC field (EEF). We distinguished and described four system states, associated with different microparticle structures. We have found that the ordered structures form due to the interactions of charges and dipoles (induced in particles by an EEF), strongly assisted by field-driven individual particle oscillations, as well as due to the local flow of ohmic current. Since the competition/cooperation among the various interactions is a function of EEF parameters, potentially a variety of structures could be formed.

We discussed in greater detail two interstate transitions involving microstructure reorganisation. In order to explain the driven gas-vertical chains state transition, we proposed a simple model of interactions, utilising the concept of a local electric field (LEF) and considering interactions between charges and dipoles. The transition was found to be independent of the EEF amplitude, and the transition frequency was found to be only on dependent material properties. We provide a simple formula for predicting the transition frequency (12), making a potentially useful tool for complex materials engineering.

The randomly oriented chains-percolation network transition was discussed only in some detail. The transition position versus EEF frequency and amplitude was found unequivocal, though a significant formation/disintegration hysteresis was observed. We found the position to be weakly dependent on the microparticles charge and we expect the network formation to be controlled by ohmic current. Thus, a description in the language of electric circuits was proposed for future development.

\section{Acknowledgments}

We would like to acknowledge the support and help from Piotr Garstecki and Tomasz Szymborski from Institute of Physical Chemistry, Polish Academy of Sciences.

\section{Appendix A: Eigenfrequency value of the charge oscillation in a particle}

In order to solve Eq. (11) and find $\omega$ corresponding to $R$ maximum for given particle/medium parameters, the eigenfrequency of the charge oscillation in the particle $\omega_{0}$ must be known first. The eigenfrequencies encountered in our experiments lie in the acoustic regime and can be estimated from the analysis of the 3-particles chain presented in Fig. 4 in the absence of EEF. Equations (4)-(8) yield then

$$
W_{p}=-\frac{1}{2} \frac{\alpha^{2}}{\left(\pi \epsilon_{0} \epsilon_{r}(2 a)^{3}\right)^{2}} E_{p}^{2}\left(\alpha+\alpha^{*}\right) .
$$


On the other hand, $-E_{p}^{2}\left(\alpha+\alpha^{*}\right) / 2=W_{p}$ in general. Thus, Eq. (13) can be rewritten in a very simple form

$$
\alpha=\pi \epsilon_{0} \epsilon_{r}(2 a)^{3} \text {. }
$$

Since for $\omega=0 \alpha=N e^{2} /\left(m_{e} \omega_{0}^{2}\right)$, the charge oscillation eigenfrequency can be determined as

$$
\omega_{0}=\frac{N e^{2}}{m_{e}} \frac{1}{\pi \epsilon_{0} \epsilon_{r}(2 a)^{3}} .
$$

\section{References}

[1] D. Helbling, I.J. Farkas, T. Viscek, Phys. Rev. Lett. 84, 1240 (2000).

[2] J. Dzubiella, G.P. Hoffmann, H. Löwen, Phys. Rev E 65, 021402 (2002).

[3] I.S. Aranson, M.V. Sapozhnikov, Phys. Rev. Lett. 92, 234301 (2004)

[4] I.S. Aranson, L.S. Tsimring, Rev. Mod. Phys. $\mathbf{7 8}$ 641 (2006).

[5] W.D. Ristenpart, I.A. Aksay, D.A. Savill, Phys. Rev. Lett. 90, 128301 (2003).

[6] F. Nadal, F. Argoul, P. Hanussen, B. Pouligny, A. Ajdari, Phys. Rev. E 65, 061409 (2002).

[7] S.H. Behrens, D.G. Grier, Phys.Rev. E 64, 050401 (2001).

[8] S.R Yeh, M. Seul, B.I. Shraiman, Nature 386, 57 (1997).

[9] I.S. Aranson, D. Blair, V.A. Kalatsky, G.W. Crabtree, W.-K. Kwok, V.M. Vinokur, U. Welp, Phys. Rev. Lett. 84, 3306 (2000).

[10] R.M. Erb, H.S. Son, B. Samanta, V.M. Rotello, B.B. Yellen, Nature 457, 999 (2009).

[11] A. Snezhko, I.S. Aranson, W.-K. Kwok, Phys. Rev. Lett. 94, 108002 (2005).

[12] O.D. Velev, K.H. Bahtt, Soft Matter 2, 738 (2006).

[13] Y. Solomentsev, M.Böhmer, J.L. Anderson, Langmuir 13, 6058 (1997).

[14] S.O. Lumsdon, E.W. Kaler, O.D. Velev, Langmuir 20 2108 (2004).

[15] M. Mittal, P.P. Lele, E.W. Kaler, E.M. Furst, J. Chem. Phys. 129, 064513 (2008).

[16] P. Dommersnes, Z. Rozynek, A. Mikkelsen, R. Castberg, K. Kjerstad, K. Hersvik, J.O. Fossum, Nat. Commun. 4, 2066 (2013).
[17] A. Mikkelsen, K. Khobaib, F.K. Eriksen, K.J. Mly, Z. Rozynek, Soft Matter 14, 5442 (2018).

[18] T. Gong, D.T. Wu, D.W.M. Marr, Langmuir 18, 10064 (2002).

[19] M.V. Sapozhnikov, Y.V. Tolmachev, I.S. Aranson, W.-K. Kwok, Phys. Rev. Lett. 90, 114301 (2003).

[20] J.C. Crocker, D.G. Grier, J. Coll. Interface Sci. 179, 298 (1996).

[21] I.S. Aranson, B. Meerson, P.V. Sasorov, V.M. Vinokur, Phys. Rev. Lett. 88, 204301 (2002).

[22] M.V. Sapozhnikov, I.S. Aranson, W.-K. Kwok, Y.V. Tolmachev, Phys. Rev. Lett. 93, 084502 (2004).

[23] W.-Q. Wu, J.-F. Liao, D.-B. Kuang, J. Energy Chem. 27, 690 (2018).

[24] D.A. Boyne, A.M. Savage, M.H. Griep, F.L. Beyer, J.A. Orlicki, Polymer 110, 250 (2017).

[25] J.A. Geldmeier, Ph.D. Thesis, Georgia Institute of Technology, 2017.

[26] A. Boltasseva, V.M. Shalaev, Metamaterials 2, 1 (2008).

[27] M.I. Aralaguppi, T.M. Aminabhavi, R.H. Balundgi, S.S. Joshi, J. Phys. Chem. 95, 5299 (1991).

[28] M. Pecovska-Gjorgjevich, A. Andonovski, J. Velevska, Maced. J. Chem. Chem. Eng. 31, 285 (2012).

[29] W.G. Scaife, C.G. Lyons, B. Bunsenges, Phys. Chem. 94, 758 (1990).

[30] M. Zientara, youtube.com/watch?v=_uxjyQ9PKQk 2019 .

[31] G.-X. Nie, W.-J. Tian, J.-P. Huang, G.Q. Gu, Chin. Phys. B 25, 067202 (2016).

[32] A. Yethiraj, A. van Blaaderen, Nature 421, 513 (2003).

[33] F. Smallenburg, H.R. Vutukuri, A. Imhof, A. van Blaaderen, M. Dijkstra, J. Phys. Condens. Matter 24, 464113 (2012).

[34] R.P. Feynman, R.B. Leighton, M. Sand, The Feynman Lectures on Physics, Addison-Wesley, 1965.

[35] W. Wen, K. Lu, Phys. Fluids 8, 2789 (1998).

[36] W. Wen, K. Lu, Phys. Fluids 9, 1826 (1997).

[37] R. Blaak, M.A. Miller, J.-P. Hansen, Europhys. Lett. 78, 26002 (2007).

[38] M.A. Miller, R. Blaak, C.N. Lumb, J.P. Hansen, J. Chem. Phys. 130, 114507 (2009).

[39] P. Ilg, E. Del Gado, Soft Matter 7, 163 (2011). 